\title{
Impact of COVID-19 on the Intestinal Microbiome
}

\author{
Carla Venegas-Borsellino ${ }^{1}$ (D) Senthilkumar Sankararaman ${ }^{2} \cdot$ Keelin Roche $^{3} \cdot$ JBracken Burns $^{3} \cdot$ Ryan Michael Landis $^{3}$
}

Accepted: 8 September 2021 / Published online: 23 November 2021

(c) The Author(s), under exclusive licence to Springer Science+Business Media, LLC, part of Springer Nature 2021

\begin{abstract}
Purpose of Review This review article aims to explore the GI changes induced by SARS-CoV-2 and how gut microbial homeostasis can influence these changes and affect the lung-gut axis and its relationship with the induction of the cytokine release syndrome in severe COVID-19 patients.

Recent Findings Coronavirus disease 2019 (COVID-19) affects not only the respiratory system but can produce multi-systemic damage. The expression of angiotensin-converting enzyme 2 (ACE-2) receptors in the gastrointestinal (GI) tract, the high prevalence of GI symptoms in severely ill COVID-19 patients, and the abnormalities described in the gut microbiome in these patients have raised concerns about the influence of GI tract as a risk factor or as a potential modulator to reduce the severity of COVID-19. Summary Understanding the mechanisms by which gut dysbiosis may influence viral transmission and disease progression in COVID-19 may help in shaping how accessible therapies, like diet modulation, can potentially help beat the devastating consequences of COVID-19.
\end{abstract}

Keywords SARS-CoV-2 · COVID-19 · Gastrointestinal manifestations $\cdot$ Microbiome $\cdot$ Brain-gut axis $\cdot$ Cytokine release syndrome $\cdot$ Probiotics

\section{Introduction}

The new SARS-CoV-2 enters through and initially affects the respiratory system, but it can generate a multiorgan dysfunction syndrome (MODS). Patients with gastrointestinal (GI) symptoms are found to have worse coronavirus disease

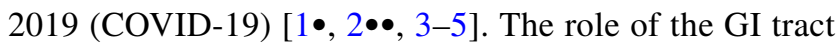
in modulating COVID-19 severity is being increasingly recognized [6]. SARS-CoV-2 may impede nutrient absorption by binding to angiotensin-converting enzyme receptors 2 (ACE2), resulting in gastroenteritis-like symptoms, and

This article is part of the Topical Collection on Gastroenterology, Critical Care, and Lifestyle Medicine

Carla Venegas-Borsellino

Venegas-Borsellino.Carla@mayo.edu

1 Department of Critical Care Medicine, Mayo Clinic, 4500 San Pablo Road Jacksonville, Jacksonville, FL 32224, USA

2 Department of Pediatrics (Pediatric Gastroenterology, UH Rainbow Babies \& Children's Hospital, Hepatology \& Nutrition), Cleveland, OH, USA

3 Department of Surgery (Trauma Surgery, Critical Care \& Acute Care Surgery), East Tennessee State University, Johnson City, TN, USA predispose an imbalance in intestinal microbial homeostasis. Chronic low-grade systemic inflammatory conditions such as advancing age, diabetes mellitus, hypertension, and obesity are associated with lower gut bacterial diversity and a disrupted gut barrier with increased permeability (leaky gut) which have been implicated in predisposing to severe SARS-CoV-2 infection $[1 \bullet, 2 \bullet \bullet, 3-5,7]$.

The gut microbiota composition and severity of acute infections, including COVID-19, have a bidirectional relationship with the lungs termed the "gut-lung axis" [7-9]. Gut dysbiosis, where the emergence of a virolent pathobiome alters signaling at the gut epithelium and generates a pro-inflammatory response, can lead to an impaired immune response and predispose to severe disease in COVID-19 [4, 10]

Understanding the mechanisms by which gut microbiome and dysbiosis may influence viral transmission and disease progression in COVID-19 may help to shape diet modulation therapies in high-risk populations and possibly reduce the devastating consequences of this pandemic. This review article aims to explore the GI changes and manifestations induced by SARS-CoV-2, its effect on gut microbiome homeostasis, and elucidate its possible effect on the lung-gut axis and its relationship with the induction of the cytokine release syndrome (CRS). 


\section{Clinical Manifestations of COVID-19 Infection in the GI Tract}

GI symptoms were underreported early in the pandemic. Huang et al. were some of the early authors to describe the SARS-CoV-2 clinical syndrome as fever, cough, and myalgias, with reported diarrhea in only one of 42 patients [11]. Other early studies out of Wuhan reported a 2-3\% incidence of GI symptoms [12]. Abdominal pain and discomfort were reported with a range of 2.2-5.8\% [13]. A recent meta-analysis reported a 15\% pool prevalence of GI manifestations which were asso-

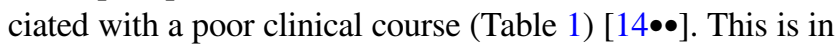
contrast with the MERS-CoV and SARS-CoV where nearly $20-25 \%$ of patients reported diarrhea or other GI complaints such as nausea or vomiting. With progression of pandemic, the prevalence of diarrhea may have been even underreported. Liang et al. argue that since there is no uniform criteria for diarrhea many hospitals or clinicians may fail to report this symptom [12].

Since the spike protein of SARS-CoV-2 enters cells through the ACE2 receptor in the small intestine, a wellknown source of intestinal inflammation, this might account for the disparity in the prevalence of diarrhea. Initially, fecal SARS-CoV-2 RNA was not thought to be directly correlated with GI symptoms, but recent evidence has debunked this theory. In Wuhan, Wei et al. found that $69 \%$ percent of patients with diarrhea had viral RNA in their stool versus $17 \%$ with only respiratory symptoms [15]. Other commonly reported symptoms were anorexia, nausea, or vomiting both at presentation and during their hospital stay [16]. Once again, these may be secondary to the virus itself or the therapies aimed at treating SARS-CoV-2.

Although diarrhea may be the most common gastrointestinal clinical symptom, transaminitis is prevalent in SARSCoV-2 patients. In early reports, around $15 \%$ of patients developed abnormal liver enzymes not explained by previ-

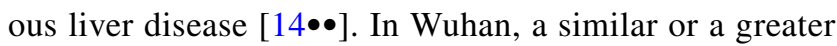
number of patients had abnormal liver enzymes, but it is

Table 1 Reported prevalence of gastrointestinal symptoms associated with SARS-CoV-2 infection

\begin{tabular}{lc}
\hline Gastrointestinal symptoms associated with SARS-CoV-2 infection \\
\hline Loss of appetite & $21 \%$ (range 1-79\%) \\
\hline Liver injury & $19 \%$ (range 1-53\%) \\
Diarrhea & $9 \%($ range $1-34 \%)$ \\
Nausea or vomiting & $6 \%$ (range 1-19\%) \\
Abdominal pain & $3 \%($ range $1-4 \%)$ \\
\hline
\end{tabular}

Ref. Mao R, et al. Manifestations and prognosis of gastrointestinal and liver involvement in patients with COVID-19: a systematic review and meta-analysis. The Lancet Gastroenterology \& Hepatology. 2020;5(7):667-78 difficult to completely attribute to SARS-CoV-2 due to the high prevalence of coexisting liver disease [17]. A review by Marjot et al. demonstrated the importance of the severity of SARS-CoV-2 in patients with cirrhosis. They found that with worsening Childs-Pugh classification, morbidity, and mortality escalated exponentially. They postulate that the cause of liver injury secondary to SARS-CoV-2 is largely multifactorial from a combination of inflammation, drug-induced (antiviral medications), hepatic congestion, and extrahepatic release of transaminases [18]. Songgozni analyzed 48 postmortem patients and found that approximately $68 \%$ had SARS-coV-2 in hepatocytes. Steatosis and microthrombosis were widely present [19].

While SARS-CoV-2 is widely seen through the GI tract, it has been difficult to directly blame the GI symptoms on SARS-CoV-2 itself. The clinical manifestations of SARS$\mathrm{CoV}-2$ are likely multifactorial in nature and the summation of viral effects via the ACE2 receptor interaction with the SARS-CoV-2 spike protein creating increased inflammation, the effects of SARS-CoV-2 pharmacotherapy therapy, or from the stress of critical illness.

\section{Shifts in the Intestinal Microbiome Associated with COVID-19}

The gut microbiome is an intricate array of thousands of species varying depending upon an individual's environmental and genetic make-up. There is a complex interplay between the gut microbiome and an individual's health, as demonstrated by studies showing associations with allergy, inflammatory conditions, and respiratory diseases [20, 21] As previously described, individuals suffering from COVID19 have several GI symptoms. Beyond those symptoms, they also have demonstrated changes in their microbiome which have broad implications in both diagnosis and treatment.

Considered the last organ discovered of the human species, the microbiome provides a unique symbiotic arrangement that has evolved over centuries. The quality and diversity of the microbiome are largely dependent on culture, diet, evolution, and location, which have been implicated in the severity of COVID-19 infection [22]. Microbiome success lies with the ability to provide autoregulation with our immune system and to allow for continued homeostasis with the living component of the microbiome. Aging, lung viral infections, and chronic illness have been associated with an alteration in the gut microbiome with an increase in the abundance of Bacteroidetes and a decrease in the abundance of Firmicutes [21, 23, 24]. Interestingly, studies performed in the murine colon have shown downregulation of ACE2 expression in the presence of Bacteroidetes species, and variable effects in the presence of Firmicutes species [25]. Hand et al. found that diabetes, cardiovascular, stroke, respiratory, 
and obesity also have significant shifts in microbiome composition [26]. This alteration may correlate with why these patient populations have had worse COVID-19 outcomes.

Dysbiosis has been implicated in multiple inflammatory and infectious diseases [20]. Several studies have demonstrated fecal viral shedding in COVID-19 patients, which persists long after respiratory symptoms have resolved. This suggests the GI tract as a site of viral replication and raises concern for fecal-oral transmission [25, 27]. Zuo et al. not only demonstrated that almost half of those infected with COVID-19 had stool positivity but also showed that those with positivity had higher levels of opportunistic bacteria including Collinsella aerofaciens, Collinsella tanakaei, Streptococcus infantis, and Morganella morganii [25]. This was further confirmed by a small study looking at 30 COVID-19 patients which demonstrated a decrease in biodiversity along with a rise in opportunistic bacteria including Streptococcus, Rothia, Veillonella, Erysipelatoclostridium, and Actinomyces [7].

An abundance of Coprobacillus, Clostridium ramosum, and Clostridium hathewayi correlated with severe COVID19 illness [25]. The abundance of beneficial Bacteroidetes species and Alistipes onderdonki were negatively correlated with severe illness and four species of Bacteroides ( $B$. dorei, B. thetaiotaomicron, B. massiliensis, and B. ovatus) exhibited negative association with stool viral load of SARSCoV-2 [25]. Many studies have explored the relationship between gut dysbiosis, severe COVID-19, and elevated inflammatory markers. Bacteroides dorei and Akkermansia muciniphila were positively correlated with IL-1 $\beta$, IL-6, and CXCL8 [28]. Similarly, IL10, TNF- $\alpha$, and chemokines such as CXCL10 and CCL2 were negatively correlated with beneficial microbiota such as Bifidobacterium adolescentis, Eubacterium rectale, and Faecalibacterium prausnitzii, and these species remained low in stool samples collected up to a month after the illness [28]. In another study involving a small group of COVID-19 patients, butyrate producers (e.g., Faecalibacterium prausnitzii, Clostridium butyricum, Clostridium leptum, and Eubacterium rectale) were reduced significantly, and opportunistic pathogens such as Enterococcus and Enterobacteriaceae were abundant in severely ill population with adverse outcome [29]. Altered gut microbiota was noted in children with Kawasaki disease both in acute and non-acute phases which further hints at the possible link between MISC in SARS-CoV-2 and dysbiosis [30].

Using 20 blood proteomic biomarkers, Gou et al. found a positive association between proteomic risk score and proinflammatory cytokines in older patients [31]. They also noted that gut microbiota was correlated with proinflammatory cytokines [31]. Further, increased levels of Lactobacillus species positively correlated with IL-10 and associated with better disease outcomes [31]. Proinflammatory species of Klebsiella, Streptococcus, and Ruminococcus gnavus correlated positively with proinflammatory cytokines and severe illness [31]. Fecal metabolomic analysis revealed deranged amino acid-related pathways providing a link between gut dysbiosis, inflammation, and disease severity [31]. Using untargeted plasma metabolomics, a recent study showed compromised gut barrier integrity and translocated products of gut microbes correlated with increased systemic inflammation and immune activation [32]. The disrupted plasma metabolome revealed a positive association between disease severity, gut permeability, and translocated products of gut microbes [32].

Given the impact of the microbiome on bacterial and viral replication, maintenance of this barrier is essential. Patients with COVID-19 infection have a decrease in beneficial bacteria and an increase in opportunistic bacteria which may be a combination of pathogenicity of the virus and host susceptibility due to chronic illness or obesity. This represents a potential area of treatment, as we have seen with the use of probiotics and fecal transplantation in other infectious diseases. While theoretically implicated, the true importance of the microbiome has not been fully elucidated. Further studies regarding composition, alteration, and therapies to restore the microbiome are needed.

\section{Dysbiosis and Altered Immune Responses in Patients with COVID-19 Disease}

Up to one fifth of patients with COVID-19 present with lifethreatening complications, including ARDS and MODS [8]. Mentioned risk factors for severe SARS-CoV-2 infection include old age, obesity, and comorbid chronic conditions $[1 \bullet, 2 \bullet \bullet, 33]$. In these susceptible populations, COVID19 can cause an overwhelming immune response which is termed as "cytokine storm syndrome (CRS)" [33-35]. Similarly, a multisystem inflammatory syndrome in children (MISC) resembling Kawasaki disease has been noted $[33,36,37]$. Aimed at the process of virus elimination and containment, this amplified immune cascade activation with hyperinflammation is characterized by a dysregulated robust secretion of proinflammatory cytokines (e.g., IL-1, IL-6, IL-17, TNF- $\alpha$, IFN- $\gamma$ ) and acute phase reactants that trigger systemic inflammation, hypercoagulation leading to ARDS and MODS [7, 34, 37-39].

In both COVID-19 patients and animal models, severe SARS-CoV-2 infection is found to be associated with low serum levels of interferons (IFN) I and III and increased chemokines and cytokines $[37,40]$. The CRS is characterized by $\mathrm{CD}^{+} \mathrm{T}$ cell predominance and frequently accompanied by a paucity of $\mathrm{CD} 8^{+} \mathrm{T}$ cells [40]. COVID-19 patients had significantly higher levels of fecal IL-18, a proinflammatory cytokine, along with higher serum levels of IL-6 and TNF- $\alpha$ when compared to healthy controls [41]. Similarly, in 
patients with severe COVID-19, proinflammatory monocytederived macrophages were found abundantly in bronchoalveolar lavage fluid [37]. MISC is associated with increased stool calprotectin signifying the association between inflammatory status and disruption in gut homeostasis [42].

The exact relationship between gut dysbiosis and systemic inflammation in CRS remains elusive; various studies have explored the possible mechanisms [43]. Decreased gut butyrate may predispose to increased binding to ACE2 with subsequent activation leading to activation of the reninangiotensin system (RAS), which may predispose to systemic vasoconstriction and systemic inflammatory response syndrome (SIRS) [1・, 44, 45]. Additionally, butyrate also increases mucin production via goblet cells and activates regulatory $\mathrm{T}$ cells, which play a vital role in attenuating cytokine production via minimizing $\mathrm{T}$ cell activation [43]. Gut dysbiosis and epithelial damage increase the expression of ACE2, increasing the SARS-CoV-2 replication in the gut and predisposing to further dissemination [6]. An imbalance in Ang II/Ang 1-7 ratio, with attenuated ACE2 protective mechanism, favors Ang II-AT ${ }_{1} \mathrm{R}$ signaling, which leads to subsequent RAS-dependent proinflammatory, prothrombotic, and vasoconstrictive events [35, 46]. Furthermore, intestinal ACE2 shedding may also predispose to gut dysbiosis [46]. The hyper-inflammation also could be contributed by the loss of modulation by Ang1-7 via Mas receptors which are normally involved in the attenuation of inflammatory response $[35,46]$. ACE2 is essential for the expression of $\mathrm{B}^{\circ} \mathrm{AT} 1$ (sodium-dependent amino acid transporter) in the intestinal epithelium, and ACE2 blockade could result in a decrease in the intestinal uptake of tryptophan, which regulates the secretion of antimicrobial peptides via the mTOR pathway, predisposing to gut dysbiosis [45, 47].

\section{Gut-Lung Axis and Its Relationship to Multiple Organ Failure}

Gastrointestinal symptoms in COVID-19 are associated with intestinal epithelial damage, impaired intestinal barrier integrity, and the altered gut microbiome [48]. This impairment can stimulate the innate and adaptive immune responses and trigger an imbalanced proinflammatory cytokines response, well described in severe cases of COVID-19. In normal individuals, immune gut homeostasis is regulated by the fine balance between pro-inflammatory pathways involving Th17 T cells and regulatory T cells, efficiently balanced by the commensal microorganisms [48]. Gut barrier disruption alters the $\mathrm{T}$ and $\mathrm{B}$ cell balance and activates the enteric inflammatory response, which signals to the circulatory system and other organs, including the lung and brain, triggering an acute respiratory distress syndrome (ARDS) and multi-organ failure described in severe
COVID-19 cases. These data reveal the association between the lung-gut-brain axis and microbiome disruption and suggest the GI tract is an important extrapulmonary site for SARS-CoV-2 infection.

Studies have shown that gut disruption can affect lung function and vice versa via bidirectional crosstalk based on messages transmitted via endotoxins and microbial metabolites [9, 49]. For example, pulmonary function can be impacted by altered immune responses regulated by gut microbiota via T cells, toll-like receptors (TLRs), neutrophils, and inflammatory cytokines [10]. Leaky gut with translocation of bacterial products (lipopolysaccharides) is implicated as one of the important factors for the progression of MODS $[4,10]$. In experimental models of sepsis and ARDS, the lung microbiome is enriched with gut-associated microbiota underpinning the shared mechanism between these diseases and gut dysbiosis [50]. Individuals most susceptible to severe COVID-19 infection are the elderly, immune-compromised, diabetic, and obese, all at risk for an unbalanced gut bacteria diversity and dysbiosis [26].

GI tract inflammation related to gut barrier dysfunction can increase the expression of ACE2 receptors and create an environment favorable to the development of infection by SARS-CoV-2 in the gut epithelium, increased gut permeability, and further spread throughout the body $[6,8]$. The importance of understanding the "gut-lung axes" in the setting of COVID-19 lies in the possibility of novel therapy or lifestyle modifications which can prevent acquisition of the disease, prevent disease progression, or risk for developing complications. It has been proposed that restoring the gut microbiota balance can be an adjuvant therapy in patients suffering from severe COVID-19.

A study of 15 patients with COVID-19 described how the altered fecal microbiome of these patients was enriched by opportunistic pathogens and depleted of beneficial commensals, and its association with the fecal presence of SARS-CoV-2 and COVID-19 severity [25]. New research is focusing on understanding the lung-gut axis interaction and altering the intestinal microbiota to reduce disease severity.

\section{Probiotics and Its Restorative Effect on Gut Microbiome in COVID-19 Patients}

Probiotics have been extensively studied in medicine over the past century [51]. There is evidence from both animal and human studies supporting antiviral and anti-inflammatory benefits, along with a connection between the "gut-lung axis" [20]. Antiviral activity is multifaceted through both direct probiotic-virus interaction intra-luminally, and stimulation of the immune system [52]. Multiple strains of LAB have been shown to reduce severity, duration, and viral titers of several viruses including cytomegalovirus, Ebola virus, and 
respiratory syncytial virus [53]. The anti-inflammatory properties have been demonstrated in both animal and human models through a reduction in IL-6 and TNF-a, pro-inflammatory cytokines, and an increase in IL-10, an anti-inflammatory marker, with administration of probiotics [53].

When discussing a role for probiotics in the treatment of COVID-19, this centers around the "gut-lung axis" [20]. The mechanism of this interaction is not completely elucidated but theorized to include antigen-presenting cells (APCs) phagocytosing microbiota and transferring to mesenteric lymph nodes and a direct lymphatic connection between the intestines and the lungs allowing for bacterial translocation [20]. The implication of all of this being that regulation and improvement of intestinal health and microbiota leads to improved respiratory function and protection from infection.

It is largely an extrapolation of this data that has been used to support the role of probiotics in the treatment of COVID19. Given the novelty of the virus in the human population there are few direct studies on COVID-19 patients, but of those that are published there is support for a dysbiosis in patients infected with COVID-19, opening a role for probiotics in the treatment of this novel and deadly virus [20].

The limited studies available on COVID-19 patients indicate intestinal dysbiosis [52]. A small report from the beginning of the pandemic indicated low levels of Lactobacillus and Bifidobacerium in COVID-19 patients (Rajput et al., 2020). Zuo et al. has shown through fecal samples almost half of patients sampled showed stool positivity for COVID-19 viral RNA which persisted long after respiratory clearance. Additionally, the authors found that patients with viral RNA had higher levels of opportunistic pathogens [25]. Several studies are currently underway to better elucidate the role of probiotics in the treatment of COVID-19. Further evidence is needed for a definitive recommendation regarding probiotics and the treatment of COVID-19, although there is biological plausibility and reasons for hope that they may play a beneficial role.

\section{Conclusions}

The clinical consequences of COVID-19 are devastating, and the GI tract role is largely underestimated. The importance of gut homeostasis and appropriate immune response toward viral infections has gained momentum during the COVID-19 pandemic due to the evidence of the types of susceptible populations. Gut microbiome composition changes has been linked to preexisting conditions associated with severe COVID-19 infection with high morbidity and mortality like old age, obesity, diabetes mellitus, and hypertension. Restoring gut homeostasis with dietary modification or microorganism supplements is a potential therapeutic target to mitigate deleterious effects in the lung-gut axis, and severe systemic inflammation and intestinal damage.
Author Contribution Carla Venegas-Borsellino, Senthilkumar Sankararaman, Keelin Roche, J Bracken Burns, and Ryan Michael Landis all had equal part in conception, literature review, and writing.

\section{Compliance with Ethical Standards}

Ethics Approval Not applicable.

Consent to Participate Not applicable.

Consent for Publication All authors consent to publication of this manuscript.

Human and Animal Rights and Informed Consent This article does not contain any studies with human or animal subjects performed by any of the authors.

Conflict of Interest The authors declare no competing interests.

\section{References}

Papers of particular interest, published recently, have been highlighted as:

- Of importance

$\bullet$ Of major importance

1. Kim HS. Do an altered gut microbiota and an associated leaky gut affect COVID-19 severity? mBio. 2021;12(1). https://doi.org/ 10.1128/mBio.03022-20. This article reviews available evidence supporting that an altered gut homeostasis may contribute to the multiorgan manifestations and complications associated to severe illness in patients with severe COVD-19 infection.

2.••Donati Zeppa S, Agostini D, Piccoli G, Stocchi V, Sestili P. Gut microbiota status in COVID-19: an unrecognized player? Front Cell Infect Microbiol. 2020;10: 576551. https://doi.org/ 10.3389/fcimb.2020.576551. This article reviews the possible biological, physiopathological, and clinical implications of gut microbiota homestoasis in the severity of COVID-19 infection and how maintaining its homeostasis could be a strategy to reduce COVID-19 virulence.

3. Wan Y, Li J, Shen L, Zou Y, Hou L, Zhu L, et al. Enteric involvement in hospitalised patients with COVID-19 outside Wuhan. Lancet Gastroenterol Hepatol. 2020;5(6):534-5. https://doi.org/10.1016/ s2468-1253(20)30118-7.

4. Zhou Q, Verne GN. Intestinal hyperpermeability: a gateway to multi-organ failure? J Clin Invest. 2018;128(11):4764-6. https:// doi.org/10.1172/jci124366.

5. Vignesh R, Swathirajan CR, Tun ZH, Rameshkumar MR, Solomon SS, Balakrishnan P. Could perturbation of gut microbiota possibly exacerbate the severity of COVID-19 via cytokine storm? Front Immunol. 2020;11: 607734. https://doi.org/10.3389/fimmu.2020.607734.

6. de Oliveira GLV, Oliveira CNS, Pinzan CF, de Salis LVV, Cardoso CRB. Microbiota modulation of the gut-lung axis in COVID-19. Front Immunol. 2021;12: 635471. https://doi.org/ 10.3389/fimmu.2021.635471.

7. Gu S, Chen Y, Wu Z, Chen Y, Gao H, Lv L, et al. Alterations of the gut microbiota in patients with coronavirus disease 2019 or H1N1 influenza. Clin Infect Dis. 2020;71(10):2669-78. https://doi.org/10.1093/cid/ciaa709. 
8. van der Lelie D, Taghavi S. COVID-19 and the gut microbiome: more than a gut feeling. mSystems. 2020;5(4). https:// doi.org/10.1128/mSystems.00453-20

9. Dumas A, Bernard L, Poquet Y, Lugo-Villarino G, Neyrolles O. The role of the lung microbiota and the gut-lung axis in respiratory infectious diseases. Cell Microbiol. 2018;20(12): e12966. https:// doi.org/10.1111/cmi.12966.

10. Chattopadhyay I, Shankar EM. SARS-CoV-2-indigenous microbiota nexus: does gut microbiota contribute to inflammation and disease severity in COVID-19? Front Cell Infect Microbiol. 2021;11: 590874. https://doi.org/10.3389/fcimb. 2021.590874.

11. Huang C, Wang Y, Li X, Ren L, Zhao J, Hu Y, et al. Clinical features of patients infected with 2019 novel coronavirus in Wuhan. China Lancet. 2020;395(10223):497-506. https://doi. org/10.1016/s0140-6736(20)30183-5.

12. Liang W, Feng Z, Rao S, Xiao C, Xue X, Lin Z, et al. Diarrhoea may be underestimated: a missing link in 2019 novel coronavirus. Gut. 2020;69(6):1141-3. https://doi.org/10.1136/gutjnl-2020-320832.

13. Wong SH, Lui RN, Sung JJ. Covid-19 and the digestive system. J Gastroenterol Hepatol. 2020;35(5):744-8. https://doi. org/10.1111/jgh.15047.

14.• Mao R, Qiu Y, He J-S, Tan J-Y, Li X-H, Liang J et al. Manifestations and prognosis of gastrointestinal and liver involvement in patients with COVID-19: a systematic review and meta-analysis. The lancet Gastroenterology \& hepatology. 2020;5(7):667-78. https://doi.org/10.1016/S2468-1253(20) 30126-6. This meta-analysis studied the epidemiological and clinical features of COVID-19, the prevalence of gastrointestinal findings and gastrointestinal comorbidities in infected patients.

15. Wei XS, Wang X, Niu YR, Ye LL, Peng WB, Wang ZH, et al. Diarrhea is associated with prolonged symptoms and viral carriage in corona virus disease 2019. Clin Gastroenterol Hepatol. 2020;18(8):1753-9.e2. https://doi.org/10.1016/j.cgh.2020.04.030.

16. Lin L, Jiang X, Zhang Z, Huang S, Zhang Z, Fang Z, et al Gastrointestinal symptoms of 95 cases with SARS-CoV-2 infection. Gut. 2020;69(6):997-1001. https://doi.org/10.1136/ gutjnl-2020-321013.

17. Chen N, Zhou M, Dong X, Qu J, Gong F, Han Y, et al. Epidemiological and clinical characteristics of 99 cases of 2019 novel coronavirus pneumonia in Wuhan, China: a descriptive study. Lancet. 2020;395(10223):507-13. https://doi.org/10. 1016/s0140-6736(20)30211-7.

18. Marjot T, Webb GJ, Barritt ASt, Moon AM, Stamataki Z, Wong VW et al. COVID-19 and liver disease: mechanistic and clinical perspectives. Nat Rev Gastroenterol Hepatol. 2021:1-17. https://doi.org/10.1038/s41575-021-00426-4.

19. Sonzogni A, Previtali G, Seghezzi M, Grazia Alessio M, Gianatti A, Licini L, et al. Liver histopathology in severe COVID 19 respiratory failure is suggestive of vascular alterations. Liver Int. 2020;40(9):2110-6. https://doi.org/10.1111/liv.14601.

20. Rajput S, Paliwal D, Naithani M, Kothari A, Meena K, Rana S. COVID-19 and gut microbiota: a potential connection. Indian J Clin Biochem. 2021:1-12. https://doi.org/10.1007/ s12291-020-00948-9.

21. Zhao W, Ho HE, Bunyavanich $\mathrm{S}$. The gut microbiome in food allergy. Ann Allergy Asthma Immunol. 2019;122(3):276-82. https://doi.org/10.1016/j.anai.2018.12.012.

22. Janda L, Mihalčin M, Šlastná M. Is a healthy microbiome responsible for lower mortality in COVID-19? Biologia (Bratisl). 2020:1-11. https://doi.org/10.2478/s11756-020-00614-8.

23. Segal JP, Mak JWY, Mullish BH, Alexander JL, Ng SC, Marchesi JR. The gut microbiome: an under-recognised contributor to the COVID-19 pandemic? Therap Adv
Gastroenterol. 2020;13:1756284820974914. https://doi.org/ $10.1177 / 1756284820974914$.

24. Calder PC. Nutrition, immunity and COVID-19. BMJ Nutr Prev Health. 2020;3(1):74-92. https://doi.org/10.1136/ bmjnph-2020-000085.

25. Zuo T, Zhang F, Lui GCY, Yeoh YK, Li AYL, Zhan H, et al. Alterations in gut microbiota of patients with COVID-19 during time of hospitalization. Gastroenterology. 2020;159(3):944-55. e8. https://doi.org/10.1053/j.gastro.2020.05.048.

26. Hand TW, Vujkovic-Cvijin I, Ridaura VK, Belkaid Y. Linking the microbiota, chronic disease, and the immune system. Trends Endocrinol Metab. 2016;27(12):831-43. https://doi.org/10. 1016/j.tem.2016.08.003.

27. Penninger JM, Grant MB, Sung JJY. The role of angiotensin converting enzyme 2 in modulating gut microbiota, intestinal inflammation, and coronavirus infection. Gastroenterology. 2021;160(1):39-46. https://doi.org/10.1053/j.gastro.2020.07.067.

28. Yeoh YK, Zuo T, Lui GC, Zhang F, Liu Q, Li AY, et al. Gut microbiota composition reflects disease severity and dysfunctional immune responses in patients with COVID19. Gut. 2021;70(4):698-706. https://doi.org/10.1136/ gutjnl-2020-323020.

29. Tang L, Gu S, Gong Y, Li B, Lu H, Li Q, et al. Clinical significance of the correlation between changes in the major intestinal bacteria species and COVID-19 severity. Engineering (Beijing). 2020;6(10):1178-84. https://doi.org/10.1016/j.eng.2020.05.013.

30. Esposito S, Polinori I, Rigante D. The gut microbiota-host partnership as a potential driver of Kawasaki syndrome. Front Pediatr. 2019;7:124. https://doi.org/10.3389/fped.2019.00124.

31. Gou W, Fu Y, Yue L, Chen G-d, Cai X, Shuai M et al. Gut microbiota may underlie the predisposition of healthy individuals to COVID-19. medRxiv. 2020. https://doi.org/10.1101/2020.04.22. 20076091.

32. Giron LB DH, Yin X, Wang H, Damra M, Goldman AR, et al. Severe COVID-19 is fueled by disrupted gut barrier integrity. medRxiv. 2020.

33. Ferreira C, Viana SD, Reis F. Gut microbiota dysbiosis-immune hyperresponse-inflammation triad in coronavirus disease 2019 (COVID-19): impact of pharmacological and nutraceutical approaches. Microorganisms. 2020;8(10). https://doi.org/10. 3390/microorganisms8101514.

34. Jose RJ, Manuel A. COVID-19 cytokine storm: the interplay between inflammation and coagulation. Lancet Respir Med. 2020;8(6):e46-7. https://doi.org/10.1016/s2213-2600(20) 30216-2.

35. Mahmudpour M, Roozbeh J, Keshavarz M, Farrokhi S, Nabipour I. COVID-19 cytokine storm: The anger of inflammation. Cytokine. 2020;133: 155151. https://doi.org/10.1016/j.cyto.2020.155151.

36. Toubiana J, Poirault C, Corsia A, Bajolle F, Fourgeaud J, Angoulvant F, et al. Kawasaki-like multisystem inflammatory syndrome in children during the covid-19 pandemic in Paris, France: prospective observational study. BMJ. 2020;369: m2094. https://doi.org/10.1136/bmj.m2094.

37. Liao M, Liu Y, Yuan J, Wen Y, Xu G, Zhao J, et al. Singlecell landscape of bronchoalveolar immune cells in patients with COVID-19. Nat Med. 2020;26(6):842-4. https://doi.org/10. 1038/s41591-020-0901-9.

38. Costela-Ruiz VJ, Illescas-Montes R, Puerta-Puerta JM, Ruiz C, Melguizo-Rodríguez L. SARS-CoV-2 infection: The role of cytokines in COVID-19 disease. Cytokine Growth Factor Rev. 2020;54:62-75. https://doi.org/10.1016/j.cytogfr.2020.06.001.

39. Dhar SK, K V, Damodar S, Gujar S, Das M. IL-6 and IL-10 as predictors of disease severity in COVID-19 patients: results from meta-analysis and regression. Heliyon. 2021;7(2). https:// doi.org/10.1016/j.heliyon.2021.e06155. 
40. Blanco-Melo D, Nilsson-Payant BE, Liu WC, Uhl S, Hoagland D, Møller R, et al. Imbalanced Host Response to SARS-CoV-2 Drives Development of COVID-19. Cell. 2020;181(5):1036-45. e9. https://doi.org/10.1016/j.cell.2020.04.026.

41. Tao W ZG, Wang X, Guo M, Zeng W, Xu Z, et al. Analysis of the intestinal microbiota in COVID-19 patients and its correlation with the inflammatory factor IL-18. Medicine in Microecology. 2020;5:100023.

42. Gonzalez Jimenez D, Velasco Rodríguez-Belvís M, Domínguez Ortega G, Segarra Cantón O, Diaz Martin JJ. Faecal calprotectin in children with multisystem inflammatory syndrome: a pilot case-control study. Acta Paediatr. 2021. https://doi.org/10.1111/ apa.15856.

43. Chen J, Hall S, Vitetta L. Altered gut microbial metabolites could mediate the effects of risk factors in Covid-19. Rev Med Virol. 2021:e2211. https://doi.org/10.1002/rmv.2211.

44. Mönkemüller K, Fry LC, Rickes S. Systemic inflammatory response and thrombosis due to alterations in the gut microbiota in COVID-19. Rev Esp Enferm Dig. 2020;112(7):584-5. https:// doi.org/10.17235/reed.2020.7297/2020.

45. Perlot T, Penninger JM. ACE2 - from the renin-angiotensin system to gut microbiota and malnutrition. Microbes Infect. 2013;15(13):866-73. https://doi.org/10.1016/j.micinf.2013.08.003.

46. Viana SD, Nunes S, Reis F. ACE2 imbalance as a key player for the poor outcomes in COVID-19 patients with age-related comorbidities - Role of gut microbiota dysbiosis. Ageing Res Rev. 2020;62: 101123. https://doi.org/10.1016/j.arr.2020.101123.

47. Gou W, Fu Y, Yue L, Chen G-d, Cai X, Shuai M et al. Gut microbiota may underlie the predisposition of healthy individuals to
COVID-19. medRxiv. 2020. https://doi.org/10.1101/2020.04.22. 20076091

48. Dhar D, Mohanty A. Gut microbiota and Covid-19- possible link and implications. Virus Res. 2020;285: 198018. https://doi.org/ 10.1016/j.virusres.2020.198018.

49. Khatiwada S, Subedi A. Lung microbiome and coronavirus disease 2019 (COVID-19): possible link and implications. Hum Microb J. 2020;17: 100073. https://doi.org/10.1016/j.humic. 2020.100073.

50. Dickson RP, Singer BH, Newstead MW, Falkowski NR, ErbDownward JR, Standiford TJ, et al. Enrichment of the lung microbiome with gut bacteria in sepsis and the acute respiratory distress syndrome. Nat Microbiol. 2016;1(10):16113. https://doi. org/10.1038/nmicrobiol.2016.113.

51. Guarner F, Khan AG, Garisch J, Eliakim R, Gangl A, Thomson A, et al. World Gastroenterology Organisation Global Guidelines: probiotics and prebiotics October 2011. J Clin Gastroenterol. 2012;46(6):468-81. https://doi.org/10.1097/ MCG.0b013e3182549092.

52. Sundararaman A, Ray M, Ravindra PV, Halami PM. Role of probiotics to combat viral infections with emphasis on COVID19. Appl Microbiol Biotechnol. 2020;104(19):8089-104. https:// doi.org/10.1007/s00253-020-10832-4.

53. Akour A. Probiotics and COVID-19: is there any link? Lett Appl Microbiol. 2020;71(3):229-34. https://doi.org/10.1111/ lam. 13334 .

Publisher's Note Springer Nature remains neutral with regard to jurisdictional claims in published maps and institutional affiliations. 